\title{
Itinerant to localized transition of $f$ electrons in the antiferromagnetic superconductor $\mathrm{UPd}_{2} \mathrm{Al}_{3}$
}

\author{
SHIN-ICHI FUJIMORI ${ }^{1 *}$, YUJI SAITOH ${ }^{1}$, TETSUO OKANE ${ }^{1}$, ATSUSHI FUJIMORI ${ }^{1,2}$, HIROSHI YAMAGAMI $^{1,3}$, \\ YOSHINORI HAGA ${ }^{4}$, ETSUJI YAMAMOTO 4 AND YOSHICHIKA ŌNUKI ${ }^{4,5}$
}

\author{
${ }^{1}$ Synchrotron Radiation Research Unit, Japan Atomic Energy Agency, Sayo, Hyogo 679-5148, Japan \\ ${ }^{2}$ Department of Physics, University of Tokyo, Hongo, Bunkyo-ku, Tokyo 113-0033, Japan \\ ${ }^{3}$ Department of Physics, Faculty of Science, Kyoto Sangyo University, Kyoto 603-8555, Japan \\ ${ }^{4}$ Advanced Science Research Center, Japan Atomic Energy Agency, Tokai, Ibaraki 319-1195, Japan \\ ${ }^{5}$ Graduate School of Science, Osaka University, Toyonaka, Osaka 560-0043, Japan \\ *e-mail: fujimori@spring8.or.jp
}

In heavy-fermion compounds, $f$ electrons show both itinerant and localized behaviour depending on the temperature ${ }^{1}$. Above a characteristic temperature, $T^{*}$, their magnetic properties can be described by completely 'localized' $f$-electron models. On the other hand, well below $T^{*}$, their Fermi surfaces can be explained by the 'itinerant' $f$-electron model ${ }^{2}$. These two models assume totally different natures of the $f$ electrons, and how they transform between these states as a function of temperature has never been understood at the level of their electronic structures. Here, we use angle-resolved photoelectron spectroscopy to study the band structure of the heavy-fermion antiferromagnetic superconductor $\mathrm{UPd}_{2} \mathrm{Al}_{3}$ below and above $T^{*}$, and find that the $f$ bands, which form the Fermi surfaces at low temperatures, are excluded from the Fermi surfaces at high temperatures. This result demonstrates how the same $f$ electrons can be both itinerant and localized.

$\mathrm{UPd}_{2} \mathrm{Al}_{3}$ shows an antiferromagnetic transition at a Neel temperature of $T_{\mathrm{N}}=14 \mathrm{~K}$ and undergoes a transition into the superconducting state at a critical temperature of $T_{\mathrm{c}}=2 \mathrm{~K}$ (ref. 3 ). Its Fermi surfaces at low temperatures were investigated by de Haas-van Alphen experiments ${ }^{4}$, and they were well explained by the itinerant $5 f$-electron model as described by band-structure calculations within the local density approximation ${ }^{5}$. At high temperatures, on the other hand, the magnetic susceptibility of $\mathrm{UPd}_{2} \mathrm{Al}_{3}$ follows the Curie-Weiss law above 50-70 K (ref. 6), suggesting almost completely localized magnetic moments at high temperatures. Therefore, $5 f$-electrons show either itinerant or localized properties depending on the temperature, and its crossover temperature $T^{*}$ is about $50-70 \mathrm{~K}$. There are two explanations for this temperature dependence of the magnetic properties. One scenario is based on the conventional dense Kondo picture where the localized $f$ moments at high temperatures are screened by a singlet coupling with the conduction electrons as the temperature decreases, and then condense into the itinerant heavy Fermi-liquid states at lower temperatures. The other is a two-component model, which assumes that one of the three $\mathrm{U} 5 f$ electrons of one uranium atom is itinerant, whereas the remaining two electrons are localized ${ }^{7}$. The former gives itinerant properties at low temperatures, whereas the latter explains the localized magnetic properties at high temperatures ${ }^{3}$. The model further conjectures that the unconventional superconductivity in $\mathrm{UPd}_{2} \mathrm{Al}_{3}$ originates from the exchange interaction between these two subsystems. To determine how the $\mathrm{U} 5 f$ electrons of $\mathrm{UPd}_{2} \mathrm{Al}_{3}$ can show both itinerant and localized properties, we have carried out angle-resolved photoelectron spectroscopy (ARPES) experiments in the soft X-ray region ${ }^{8,9}$ (SX-ARPES) at temperatures well below and above $T^{*}(20$ and $100 \mathrm{~K})$, and observed a remarkable temperature dependence of its band structure.

Figure 1a shows the angle-integrated photoemission (AIPES) spectra of $\mathrm{UPd}_{2} \mathrm{Al}_{3}$ measured with $h v=400$ and $800 \mathrm{eV}$ at $20 \mathrm{~K}$. In this photon energy range, the contributions from the $\mathrm{U} 5 f$ and $\mathrm{Pd} 4 d$ states are dominant, and those from the $\mathrm{Al} 3 s, 3 p$ and $\mathrm{U} 6 d$ states are one to two orders of magnitude smaller ${ }^{10}$. In Fig. 1a, we have also indicated the partial density of states (DOS) of the $\mathrm{U} 5 f$ and the Pd $4 d$ states obtained by a relativistic bandstructure calculation within the local density approximation ${ }^{11}$. Comparison with the calculation suggests that the $\mathrm{Pd} 4 d$ states are distributed mainly below the binding energy $E_{\mathrm{B}}=3 \mathrm{eV}$ and the $\mathrm{U} 5 f$ states around the Fermi energy, $E_{\mathrm{F}}$. To extract contributions from the U $5 f$ states, we have subtracted the spectrum measured at $h v=400 \mathrm{eV}$ from that measured at $h v=800 \mathrm{eV}$, as shown in Fig. $1 \mathrm{~b}$, because the photoemission cross-section of the $\mathrm{U} 5 f$ states relative to that of the $\mathrm{Pd} 4 d$ states increases by a factor of two in going from $h v=400 \mathrm{eV}$ to $h v=800 \mathrm{eV}$ (ref. 10). The difference spectrum representing the $\mathrm{U} 5 f$ distribution, as indicated by the blue line in Fig. 1b, shows a sharp peak just below $E_{\mathrm{F}}$, and a broad satellite structure extending to around $E_{\mathrm{B}}=1.5 \mathrm{eV}$. In Fig. 1b, we compare the $\mathrm{U} 5 f$ difference spectrum (blue line) with the calculated $U 5 f$ DOS broadened by the gaussian function representing the instrumental resolution (yellow line). As the observed sharp peak structure located around $E_{\mathrm{F}}$ corresponds to the calculated DOS, this peak should originate from the itinerant quasiparticle bands. On the other hand, the broad 
a

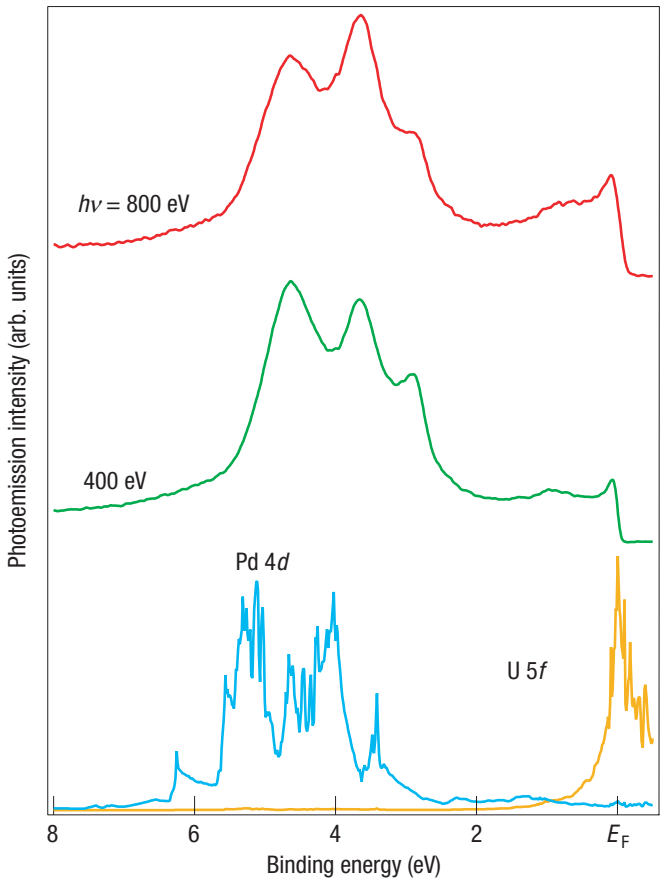

b

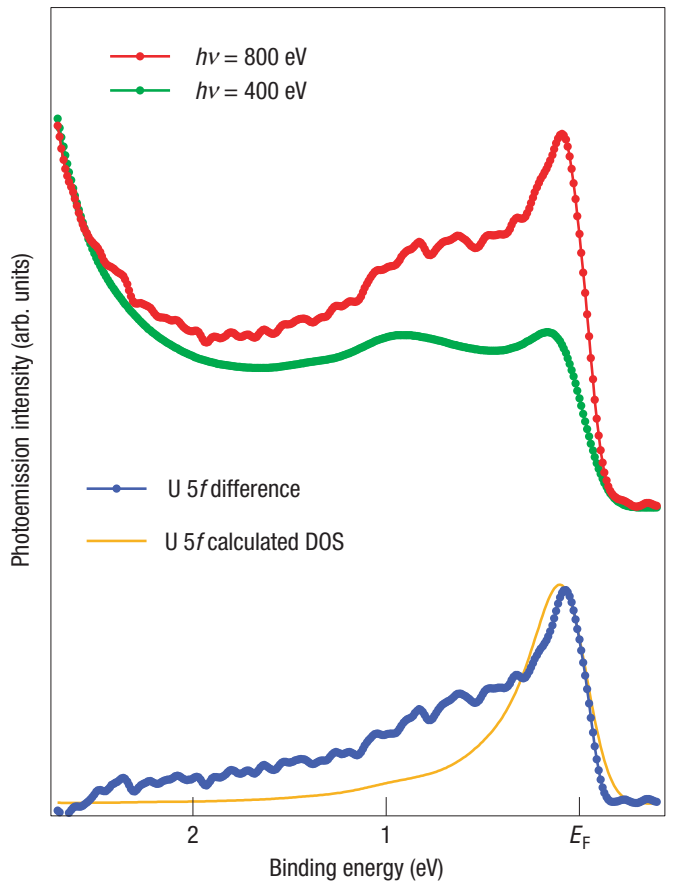

Figure 1 AIPES spectra of $\mathrm{UPd}_{2} \mathrm{Al}_{3}$. a, The spectra measured at $h v=400$ and $800 \mathrm{eV}$, together with the calculated Pd $4 d$ and $\mathrm{U} 5 f$ density of states. $\mathbf{b}$, The procedure for deriving the experimental U $5 f$ partial DOS. The spectrum measured at $h v=400 \mathrm{eV}$ has been subtracted from that measured at $h v=800 \mathrm{eV}$. The spectrum measured at $h v=400 \mathrm{eV}$ has been broadened to simulate the energy resolution of the spectrum measured at $h v=800 \mathrm{eV}$. Both spectra have been normalized to match the tail of the $\mathrm{Pd} 4 d$ states located within $E_{\mathrm{B}}=2-3 \mathrm{eV}$ with each other. The obtained U $5 f$ partial DOS is shown and is compared with the calculated U $5 f$ partial DOS.

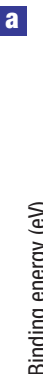

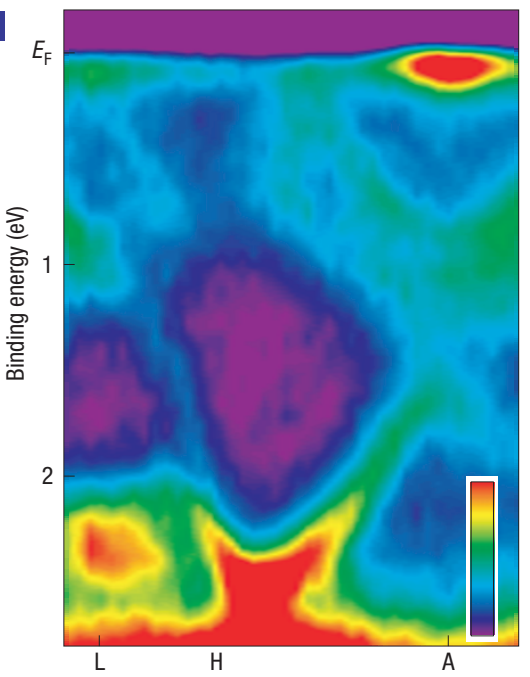

b

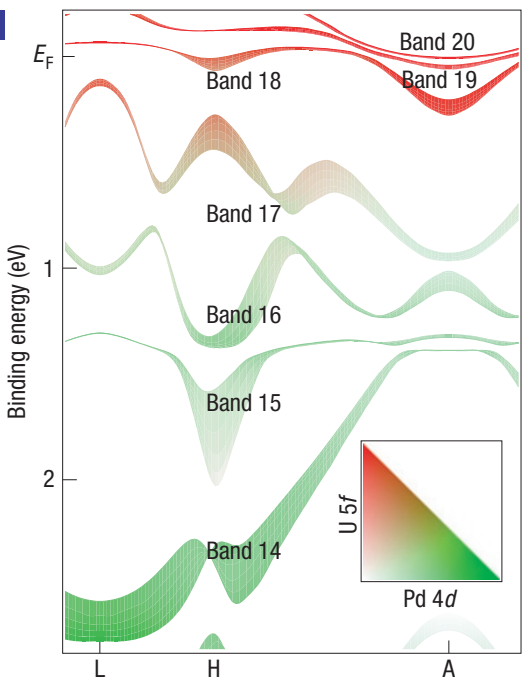

$\mathbf{G}$

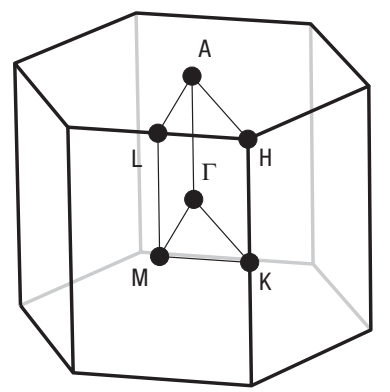

d

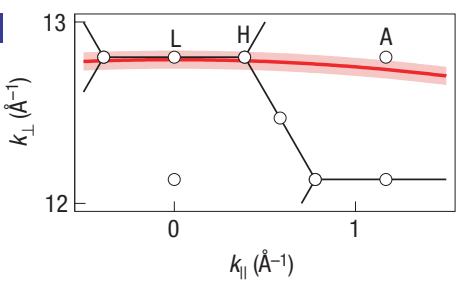

Figure 2 SX-ARPES spectra of $\mathrm{UPd}_{2} \mathrm{Al}_{3}$. a, The density plot of the SX-ARPES spectra measured at $20 \mathrm{~K}$. b. The calculated energy dispersions to be compared with the experiment. Contributions from the U $5 f$ and Pd $4 d$ states in each band are also indicated on the colour scale. c, The hexagonal Brillouin zone of $U \mathrm{Pd}_{2} \mathrm{Al}_{3}$ in a paramagnetic phase. $\mathbf{d}$, The position of the ARPES scan. The red curve represents the momentum position of the ARPES cut with $h v=595 \mathrm{eV}$. $\theta=0^{\circ}$ corresponds to the $\mathrm{L}$ point and $\theta=5.4^{\circ}$ to the A point. The shaded area indicates the momentum broadening for the $k_{\perp}$ direction owing to the finite electron escape depth.

satellite structure on the deeper binding energy side $\left(E_{\mathrm{B}}>0.5 \mathrm{eV}\right)$ cannot be explained by the band-structure calculation, suggesting that it might originate from the electron-correlation effect. In previous work ${ }^{12}$, it was proposed that this broad structure is the contribution from the completely localized $\mathrm{U} 5 f^{2}$ subsystem. If this is the case, there should be only little energy dispersion, as has been observed in the ARPES spectra of the localized U $5 f$ system $\mathrm{UPd}_{3}($ ref. 13). 

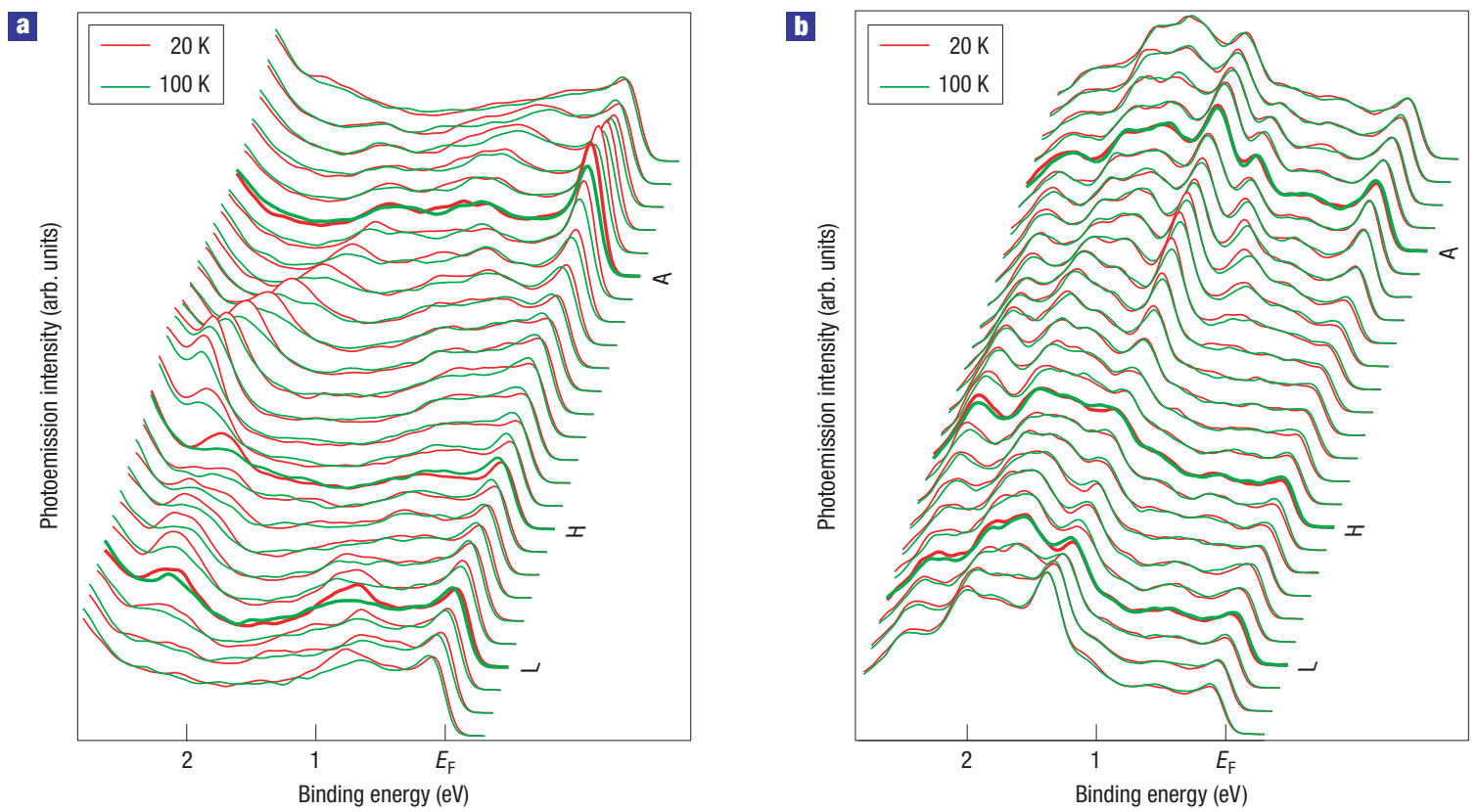

Figure 3 Temperature dependence of ARPES spectra. a, ARPES spectra of $\mathrm{UPd}_{2} \mathrm{Al}_{3}$ measured at 20 and $100 \mathrm{~K}$. b, ARPES spectra of UNi $\mathrm{Al}_{3}$ measured at 20 and $100 \mathrm{~K}$. The temperature dependencies were observed in $\mathrm{UPd}_{2} \mathrm{Al}_{3}$, but not in $\mathrm{UNi}_{2} \mathrm{Al}_{3}$.

Figure 2c,d shows the hexagonal Brillouin zone of $\mathrm{UPd}_{2} \mathrm{Al}_{3}$ and the momentum position of the present ARPES cut measured at $h v=595 \mathrm{eV}$ by the red curve. The present ARPES cut traces near the $\mathrm{L}-\mathrm{H}-\mathrm{A}$ high-symmetry line. In Fig. 2a, we show the density plot of the ARPES spectra measured at $20 \mathrm{~K}$. In the near- $E_{\mathrm{F}}$ part of the spectra, narrow quasiparticle bands of $U 5 f$-state origin can be observed. On the other hand, some strongly dispersive bands can clearly be observed in the energy region of $E_{\mathrm{B}}=0.5-1.0 \mathrm{eV}$, where the broad satellite structure is observed in the U $5 f$ AIPES spectra. Therefore, the origin of the broad satellite structure in the AIPES spectra is the contribution not from less-dispersive localized $U 5 f$ states but from strongly dispersive bands with a finite contribution from $U 5 f$ states. In Fig. $2 \mathrm{~b}$, we show the results of the energy band calculation for the $\mathrm{L}-\mathrm{H}-\mathrm{A}$ direction, in which all of the $U 5 f$ electrons are treated as being itinerant. It is shown that some calculated bands correspond to the experimental bands. In particular, bands with large contributions from $\mathrm{Pd} 4 d$ states located at $E_{\mathrm{B}}>1.0 \mathrm{eV}$ (band 14 and part of bands 15 and 16) agree with the ARPES spectra. On the other hand, the calculated bands with a large contribution from the $\mathrm{U} 5 f$ states are different from the experimentally measured bands. For example, band 17 does not match with the experiment in the energy region of $0.2-0.5 \mathrm{eV}$. In the near- $E_{\mathrm{F}}$ part of the spectra, the electron-like Fermi-surface feature around the A point shows agreement between experiment and calculation. On the other hand, a weak peak structure also remains at $E_{\mathrm{F}}$ at other momentum positions, in disagreement with the calculation where the $f$-derived bands are far from $E_{\mathrm{F}}$ except for around the $\mathrm{H}$ and A points. Therefore, $\mathrm{U} 5 f$ states are strongly renormalized in the experiment, and they could not be well explained by the band-structure calculation.

Figure 3a shows a comparison of the ARPES spectra of $\mathrm{UPd}_{2} \mathrm{Al}_{3}$ measured at 20 and $100 \mathrm{~K}$, which are sufficiently lower and higher than $T^{*}(\sim 50 \mathrm{~K})$. Although the main structures of the spectra are very similar at 20 and $100 \mathrm{~K}$, differences between them are clearly observed. The quasiparticle peaks located around $E_{\mathrm{F}}$ show strong momentum dependence. When increasing the temperature

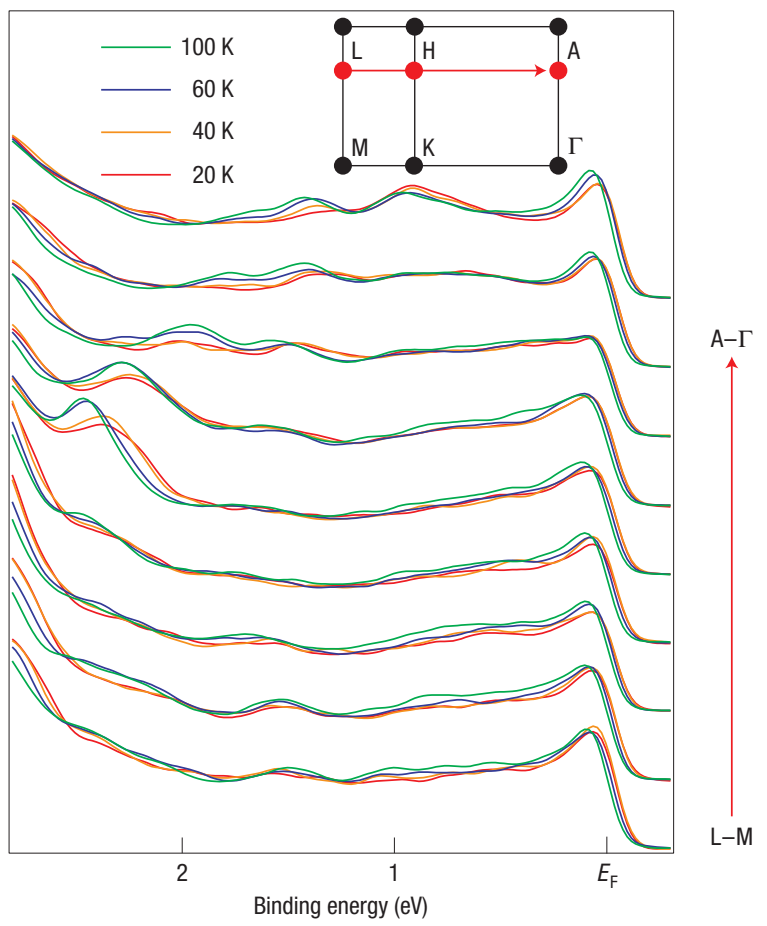

Figure 4 Detailed temperature dependence of ARPES spectra. ARPES spectra of $\mathrm{UPd}_{2} \mathrm{Al}_{3}$ measured at $100,60,40$ and $20 \mathrm{~K}$. The position of the ARPES scan is indicated in the inset.

from 20 to $100 \mathrm{~K}$, their intensities are decreased around the $\mathrm{H}$ point, whereas they are increased around the A point. Meanwhile, the position of the quasiparticle peak uniformly moves towards slightly higher binding energy sides as the temperature is increased. 

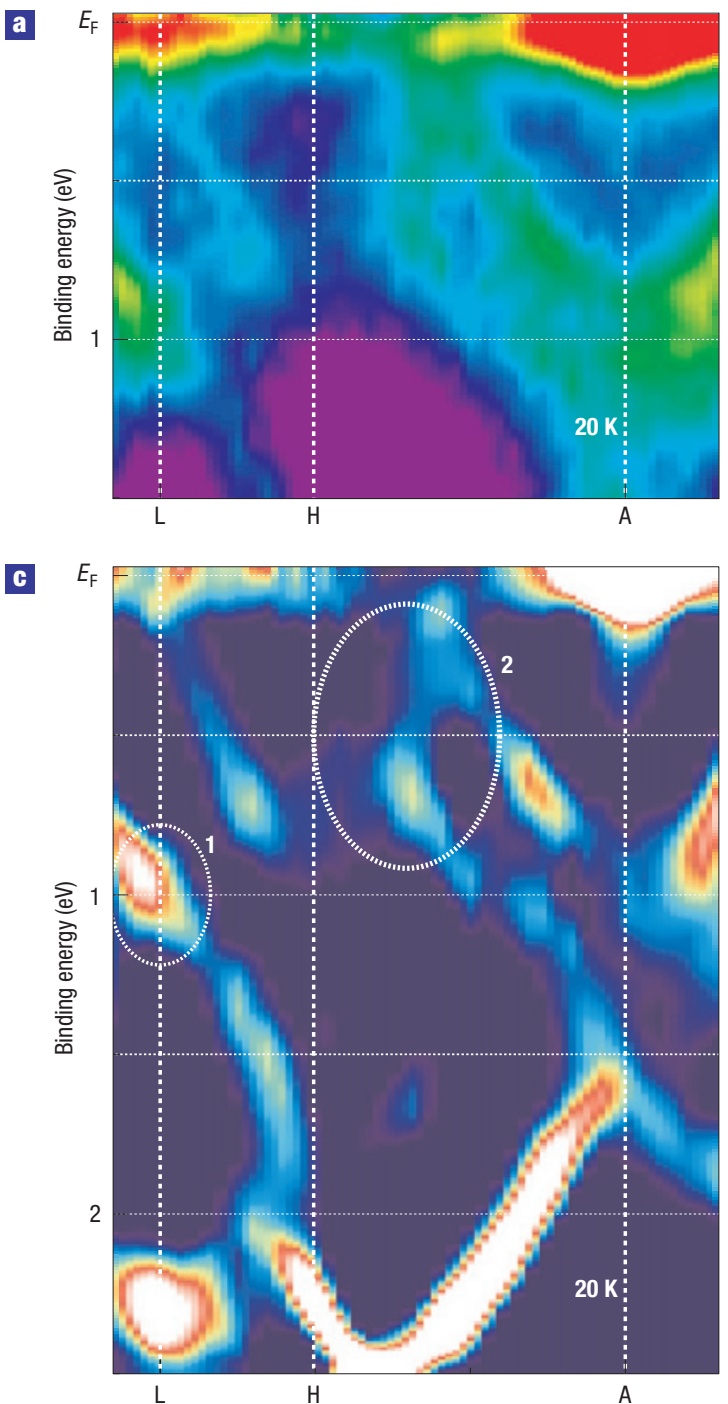

b

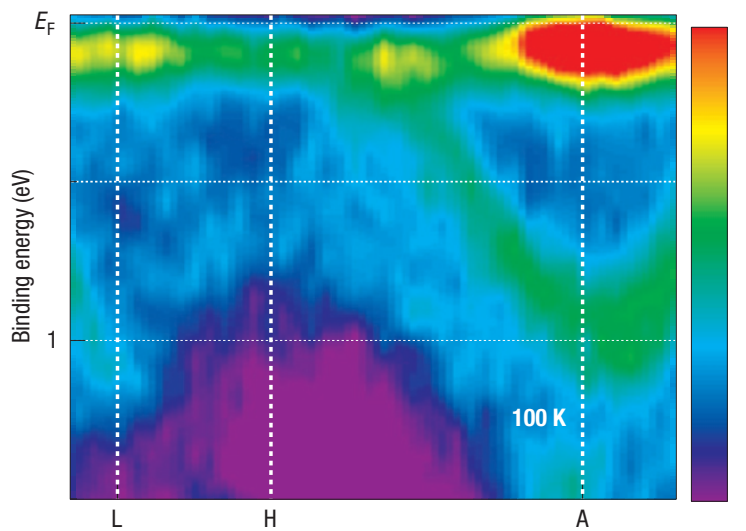

d

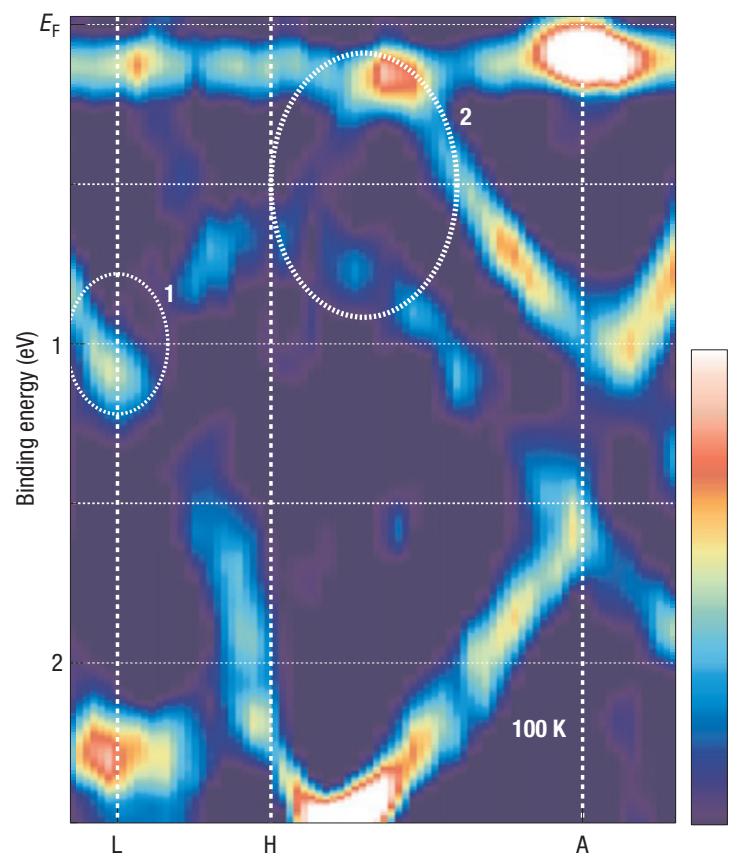

Figure $\mathbf{5}$ Measured band structures of $\mathrm{UPd}_{2} \mathbf{A l}_{3}$. a,b, ARPES spectra divided by the convoluted Fermi-Dirac function. c,d, Band structure derived by adding the second derivatives of the energy distribution curves and the momentum distribution curves in an appropriate ratio. The higher intensity part corresponds to the peak position in the ARPES spectra. The outlined areas indicate the positions of changes in the band structure in the higher binding energy sides.

Changes in spectral functions were also observed at higher binding energy sides. However, here we note that the changes in $E_{\mathrm{B}}>1.5 \mathrm{eV}$ are mainly changes in the peak intensity, and their positions are not changed, as described below. This suggests that they are not intrinsic changes in the electronic structures. We have further measured the temperature dependence of the ARPES spectra of $\mathrm{UNi}_{2} \mathrm{Al}_{3}$, whose characteristic temperature is higher than $300 \mathrm{~K}$ (ref. 14). Figure $3 \mathrm{~b}$ shows a comparison of the ARPES spectra of $\mathrm{UNi}_{2} \mathrm{Al}_{3}$ measured at 20 and $100 \mathrm{~K}$. It is clear that the spectra are not different at these two temperatures. Therefore, we conclude that the temperature dependence of the ARPES spectra of $\mathrm{UPd}_{2} \mathrm{Al}_{3}$ at around $E_{\mathrm{B}}=E_{\mathrm{F}}-1.5 \mathrm{eV}$ originates from the changes in the electronic structure of $\mathrm{UPd}_{2} \mathrm{Al}_{3}$ below and above $T^{*}$.

To confirm the robustness of the temperature dependence and to study the temperature evolution of the spectra in more detail, we measured the ARPES spectra of $\mathrm{UPd}_{2} \mathrm{Al}_{3}$ at different positions in the Brillouin zone at smaller temperature intervals. Figure 4 shows the temperature evolution of the spectra, measured at $100,60,40$ and $20 \mathrm{~K}$. The spectra show similar temperature dependencies to those observed in Fig. 3a. In the near- $E_{\mathrm{F}}$ part of the spectra, the peak position moves towards higher binding energies as the temperature increases. On the higher binding energy side, there are also substantial temperature dependencies. The spectra measured at 100 and $60 \mathrm{~K}$ show very similar structures, as do those measured at 40 and $20 \mathrm{~K}$, whereas there are considerable differences between the two temperature ranges. Therefore, this temperature dependence with an abrupt change between 40 and $60 \mathrm{~K}$ is a robust feature of the ARPES spectra of $\mathrm{UPd}_{2} \mathrm{Al}_{3}$.

We further analyse the temperature dependence of the ARPES spectra shown in Fig. 3a. In Fig. 5a,b, we show the ARPES spectra measured at 20 and $100 \mathrm{~K}$, which are divided by the FermiDirac function convoluted with the gaussian function to reveal the behaviour of the quasiparticle bands in the vicinity of $E_{\mathrm{F}}$. The temperature dependence of the quasiparticle bands is clear in these figures. Furthermore, we have taken the second derivatives of the ARPES spectra to identify the peak positions in these spectra, as shown in Fig. $5 \mathrm{c}(20 \mathrm{~K})$ and Fig. $5 \mathrm{~d}(100 \mathrm{~K})$. In these figures, the bright parts correspond to the peaks in the ARPES spectra. At $20 \mathrm{~K}$, 
the quasiparticle bands below $E_{\mathrm{F}}$ disperse to above $E_{\mathrm{F}}$, suggesting that they participate in the formation of the Fermi surfaces. On the other hand, at $100 \mathrm{~K}$, they move towards the higher binding energy side, and form less dispersive bands around $E_{\mathrm{B}}=0.1 \mathrm{eV}$. These changes imply that the quasiparticle bands of $U 5 f$ origin at low temperatures, which form the heavy Fermi-liquid states, are excluded from the Fermi surfaces at high temperatures. This behaviour of the quasiparticle bands is consistent with optical conductivity data, where the renormalized Drude peak and the hybridization gap due to the formation of the heavy quasiparticle bands are observed at $T<50 \mathrm{~K}$ whereas the usual metallic behaviour with less Drude weight is observed at $T>50 \mathrm{~K}$ (ref. 15). The usual metallic behaviour at $T>50 \mathrm{~K}$ suggests that non$f$ bands form Fermi surfaces at high temperatures. However, their contributions cannot be clearly observed in these spectra as the lower energy parts of the spectra are dominated by strong U $5 f$ contributions. These changes are consistent with the dense Kondo scenario, where the heavy quasiparticle bands are formed by hybridization between the flat renormalized $f$ level located around $E_{\mathrm{F}}$ and dispersive non- $f$ bands ${ }^{16}$. On the other hand, the temperature-induced changes in the deeper binding energy region $\left(E_{\mathrm{B}}<1 \mathrm{eV}\right)$ can also be discerned in these figures. For example, around the areas designated as 1 in Fig. $5 c, d$, the structure located at $E_{\mathrm{B}}=1 \mathrm{eV}$ moves towards $E_{\mathrm{B}}=1.1 \mathrm{eV}$ in going from 20 to $100 \mathrm{~K}$. In addition, the complicated band structures indicated in the areas designated as 2 also have significant temperature dependencies. This is in contrast to the changes at $E_{\mathrm{B}}>1.2 \mathrm{eV}$, where the band structure was essentially unchanged at the two temperatures. Thus, the transition is accompanied by drastic changes in the band structure of an energy of the order of $1 \mathrm{eV}$ $\left(\sim 10^{4} \mathrm{~K}\right)$, which is not expected in the theoretical prediction of the periodic Anderson model ${ }^{17}$. Therefore, the global nature of $\mathrm{U} 5 f$ electrons of $\mathrm{UPd}_{2} \mathrm{Al}_{3}$ including the temperature dependence of the band structures cannot be explained by any of the current theories, and the present results provide a new scheme to describe the itinerant and localized nature of $f$ electrons as well as the coexistence of magnetism and superconductivity in heavy-fermion compounds. Finally, we comment on the relationship between our study and other ARPES studies on heavy-fermion compounds. Although there have been several ARPES studies on heavy-fermion compounds, energy dispersions were not always clearly observed for the $f$ levels ${ }^{18,19}$. One possible reason is that the measurements were done above $T^{*}$, and therefore the coherence between the $f$ sites was not developed in those experiments. In addition, those ARPES experiments were done with low-energy photons, and contributions from localized surface layers could not be ruled out. Therefore, we propose that low temperatures and reduced surface contributions are indispensable for detecting the energy dispersion of $f$-electron states in heavy-fermion compounds.

\section{METHODS}

\section{PHOTOEMISSION EXPERIMENT AND DATA ANALYSIS}

Photoemission experiments were carried out at the soft X-ray beamline BL23SU of SPring-8 (ref. 20) using a photoemission spectrometer equipped with a Gammadata-Scienta SES-2002 electron analyser. The AIPES spectra were measured using the angle-integrated mode of the spectrometer. The energy resolutions were $160 \mathrm{meV}(h \nu=800 \mathrm{eV})$ and $80 \mathrm{meV}(h \nu=400 \mathrm{eV})$. The energy and angular resolution of the SX-ARPES experiments were set to $120 \mathrm{meV}$ and $0.16^{\circ}$ (corresponding to $0.036 \AA^{-1}$ ) respectively for a photon energy of $595 \mathrm{eV}$ to obtain a reasonable count rate. The samples were cooled using a closed-cycle He refrigerator. The sample temperature was measured using a chromel-AuFe thermocouple mounted close to the sample. The base pressure of the spectrometer was better than $2 \times 10^{-8} \mathrm{~Pa}$. The position of the Fermi level was determined by the position of that of in situ evaporated gold film. The measured momentum positions were determined with a free-electron final-state model by taking the photon momentum into consideration. The momentum of the electron perpendicular to the surface with the free-electron final-state model is given by

$$
k_{\perp}=\sqrt{\frac{2 m}{\hbar^{2}}\left(E_{\mathrm{kin}} \cos ^{2} \theta+V_{0}\right)}-k_{\perp \text { photon }},
$$

where $m$ is the electron mass, $E_{\text {kin }}$ is the kinetic energy of the photoelectron, $V_{0}$ is the inner potential, $\theta$ is the emission angle of the photoelectron relative to the surface normal and $k_{\perp \text { photon }}$ is the momentum of the incident photon perpendicular to the surface. We assumed that the inner potential was $V_{0}=12 \mathrm{eV}$, which is a typical value for heavy-fermion compounds ${ }^{19}$. To check the validity of the temperature dependencies of the ARPES spectra, we carried out different ARPES scans for a different $\mathrm{UPd}_{2} \mathrm{Al}_{3}$ single crystal, and confirmed that the temperature dependence of the ARPES spectra is reproducible. The sample orientation was measured ex situ using Laue photography. The image plots shown in Fig. $5 c$,d were obtained by adding the second derivative of the energy distribution curves with an appropriate weight to make the band images most clearly visible.

\section{SAMPLE PREPARATION}

The samples were characterized by $\mathrm{X}$-ray diffraction. Lattice parameters at room temperature were determined to be $a=5.368 \AA$ and $c=4.189 \AA$, in good agreement with previous studies ${ }^{21}$. The occupancy parameter at the $\mathrm{Pd}$ and $\mathrm{Al}$ sites was also refined to be $100 \%$ within an experimental accuracy of a few per cent. The direction of the crystal axes were determined using the Laue method. A clean sample surface was obtained by cleaving the sample in situ.

Received 4 February 2007; accepted 1 June 2007; published 1 July 2007.

References

1. Fulde, P. Electron Correlations in Molecules and Solids 2nd edn, Ch. 13, 309-311 (Springer, Berlin, 1993).

2. Haga, Y. et al. Fermi surface study on uranium-based intermetallic compounds. J. Nucl. Sci. Technol. Suppl. 3, 56-62 (2002).

3. Geibel, C. et al. Heavy-fermion superconductivity at $T_{\mathrm{C}}=2 \mathrm{~K}$ in the antiferromagnet $\mathrm{UPd}_{2} \mathrm{Al}_{3}$. Z. Phys. B 84, 1-2 (1991).

4. Inada, Y. et al. Fermi surface and de Haas-van Alphen oscillation in both the normal and superconducting mixed states of $\mathrm{UPd}_{2} \mathrm{Al}_{3}$. J. Phys. Soc. Jpn 68, 3643-3654 (1999).

5. Knöpfle, K. et al. The Fermi surface of $\mathrm{UPd}_{2} \mathrm{Al}_{3}$. J. Phys. Condens. Matter 8, 901-909 (1996)

6. Grauel, A. et al. Tetravalency and magnetic phase diagram in the heavy fermion superconductor $\mathrm{UPd}_{2} \mathrm{Al}_{3}$. Phys. Rev. B 46, 5818-5821 (1993).

7. Sato, N. K. et al. Strong coupling between local moments and superconducting 'heavy' electrons in $\mathrm{UPd}_{2} \mathrm{Al}_{3}$. Nature 410, 340-343 (2001).

8. Sekiyama, A. et al. Technique for bulk Fermiology by photoemission applied to layered ruthenates. Phys. Rev. B 70, 060506 (2005)

9. Fujimori, S.-I. et al. Itinerant band states in the layered compound $\mathrm{UFeGa}_{5}$ observed by soft X-ray angle-resolved photoemission spectroscopy. Phys. Rev. B 73, 125109 (2006).

10. Yeh, J. J. \& Lindau, I. Atomic subshell photoionization cross sections and asymmetry parameters $1 \leq Z \leq 103$. At. Data Nucl. Data Tables 32, 1-155 (1985).

11. Yamagami, H. All-electron spin-polarized relativistic linearized APW method: Electronic and magnetic properties of BCC Fe, HCP Gd and uranium monochalcogenides. J. Phys. Soc. Jpn 67, 3176-3190 (1998)

12. Takahashi, T. et al. Dual character of $5 f$ electrons in $\mathrm{UPd}_{2} \mathrm{Al}_{3}$ observed by high-resolution photoemission spectroscopy. J. Phys. Soc. Jpn 65, 156-159 (1996).

13. Ito, T. et al. Band structure of $\mathrm{UPd}_{3}$ studied by ultrahigh-resolution angle-resolved photoemission spectroscopy. Phys. Rev. B 66, 245110-245116 (2002).

14. Kyogaku, M. et al. Superconductivity and magnetism of the new heavy fermion superconductors $\mathrm{UM}_{2} \mathrm{Al}_{3}(\mathrm{M}=\mathrm{Ni}$, Pd). J. Phys. Soc. Jpn 61, 2660-2664 (1992).

15. Dressel, M. et al. Nature of heavy quasiparticles in magnetically ordered heavy fermions $\mathrm{UPd}_{2} \mathrm{Al}_{3}$ and $\mathrm{UPt}_{3}$. Phys. Rev. Lett. 88, 186404 (2002).

16. Zwicknagl, G. Quasi-particles in heavy fermion systems. Adv. Phys. 41, 203-302 (1992).

17. Tahvildar-Zadeh, A. N., Jarrell, M. \& Freericks, J. K. Low temperature coherence in the periodic Anderson model: Predictions for photoemission of heavy fermion. Phys. Rev. Lett. 80, 5168-5171 (1998)

18. Ito, T. et al. High-resolution angle-resolved photoemission study of the heavy-fermion superconductor $\mathrm{UPt}_{3}$. Phys. Rev. B 59, 8923-8929 (1999).

19. Denlinger, J. D. et al. Comparative study of the electronic structure of $\mathrm{XRu}_{2} \mathrm{Si}_{2}$ : Probing the Anderson lattice. J. Electron Spectrosc. Relat. Phenom. 117-118, 347-369 (2001).

20. Saitoh, Y. et al. First results from the actinide science beamline BL23SU at SPring-8. Nucl. Instrum. Methods Phys. Res. A 474, 253-258 (2001).

21. Haga, Y. et al. Single crystal growth, normal and superconducting properties of $\mathrm{UPd}_{2} \mathrm{Al}_{3}$. J. Phys. Soc. Ipn 65, 3646-3653 (1996).

\section{Acknowledgements}

We would like to acknowledge N. K. Sato, K. Miyake, N. Aso, G. Zwicknagl, G. H. Lander and A. Chainani for stimulating discussions and comments. This work was financially supported by a Grant-in-Aid for Scientific Research from the Ministry of Education, Culture, Sports, Science, and Technology Japan under contract No.15740226 and REIMEI Research Resources from the Japan Atomic Energy Agency.

Correspondence and requests for materials should be addressed to S.-I.F.

Competing financial interests

The authors declare no competing financial interests 